MATHEMATICS OF COMPUTATION

Volume 72, Number 241, Pages 499-519

S 0025-5718(02)01418-7

Article electronically published on May 2, 2002

\title{
NEW QUADRATIC POLYNOMIALS WITH HIGH DENSITIES OF PRIME VALUES
}

\author{
MICHAEL J. JACOBSON, JR. AND HUGH C. WILLIAMS
}

\begin{abstract}
Hardy and Littlewood's Conjecture F implies that the asymptotic density of prime values of the polynomials $f_{A}(x)=x^{2}+x+A, A \in \mathbb{Z}$, is related to the discriminant $\Delta=1-4 A$ of $f_{A}(x)$ via a quantity $C(\Delta)$. The larger $C(\Delta)$ is, the higher the asymptotic density of prime values for any quadratic polynomial of discriminant $\Delta$. A technique of Bach allows one to estimate $C(\Delta)$ accurately for any $\Delta<0$, given the class number of the imaginary quadratic order with discriminant $\Delta$, and for any $\Delta>0$ given the class number and regulator of the real quadratic order with discriminant $\Delta$. The Manitoba Scalable Sieve Unit (MSSU) has shown us how to rapidly generate many discriminants $\Delta$ for which $C(\Delta)$ is potentially large, and new methods for evaluating class numbers and regulators of quadratic orders allow us to compute accurate estimates of $C(\Delta)$ efficiently, even for values of $\Delta$ with as many as 70 decimal digits. Using these methods, we were able to find a number of discriminants for which, under the assumption of the Extended Riemann Hypothesis, $C(\Delta)$ is larger than any previously known examples.
\end{abstract}

\section{INTRODUCTION}

Consider the polynomial $f(x)=a x^{2}+b x+c$. If $p \mid f(X)$ for some $X \in \mathbb{Z}$, then $\Delta=b^{2}-4 a c$, the discriminant of $f(x)$, must be a square modulo $p$. Thus, if $\Delta$ is not a square modulo many primes $p$, we expect $f(x)$ to take on many prime values asymptotically. Hardy and Littlewood formalized this phenomenon as Conjecture $\mathrm{F}$ in [10]. If $\pi_{f}(n)$ denotes the number of prime values assumed by $f(X)$ for $X=0,1, \ldots, n$, then their conjecture can be given as follows:

Conjecture (F). Let $a>0, b, c$ be integers such that $\operatorname{gcd}(a, b, c)=1, \Delta=b^{2}-4 a c$ is not a square and $a+b, c$ are not both even. Then there are infinitely many primes of the form $f(x)$, and

$$
\pi_{f}(n) \sim \varepsilon C_{f} \operatorname{Li}(n),
$$

where

$$
\begin{gathered}
\operatorname{Li}(n)=\int_{2}^{n} \frac{d x}{\log x}, \\
\varepsilon= \begin{cases}\frac{1}{2} & \text { when } 2 \nmid a+b, \\
1 & \text { otherwise },\end{cases}
\end{gathered}
$$

Received by the editor September 8, 1999 and, in revised form, February 28, 2001.

2000 Mathematics Subject Classification. Primary 11R11, 11R29, 11Y40; Secondary 11Y16.

Key words and phrases. Prime-generating quadratic polynomial, quadratic order, class group.

(C)2002 American Mathematical Society 
and

$$
C_{f}=\prod_{\substack{p>2 \\ p \mid(a, b)}} \frac{p}{p-1} \prod_{\substack{p>2 \\ p \nmid a}}\left(1-\frac{\left(\frac{\Delta}{p}\right)}{p-1}\right) .
$$

The products in the expression for $C_{f}$ are taken over the primes only, and $\left(\frac{\Delta}{p}\right)$ denotes the Legendre symbol. Note here that $\varepsilon C_{f}$ is what really determines the density of prime values assumed by $f$, since $\operatorname{Li}(n)$ is a function of $n$ only. The larger $\varepsilon C_{f}$ is, the higher the asymptotic density of prime values for any quadratic polynomial of discriminant $\Delta$.

We restrict ourselves to polynomials of the form $f_{A}(x)=x^{2}+x+A$. If we denote by $P_{A}(n)$ the number of prime values assumed by $f_{A}(x)$ for $0 \leq x \leq n$, then for these polynomials we have the following simplified form of Conjecture $\mathrm{F}$ :

$$
P_{A}(n) \sim C(\Delta) L_{A}(n),
$$

where

$$
L_{A}(n)=2 \int_{0}^{n} \frac{d x}{\log f_{A}(x)}
$$

and

$$
C(\Delta)=\prod_{p \geq 3} 1-\frac{\left(\frac{\Delta}{p}\right)}{p-1} .
$$

Here $\Delta=1-4 A$.

The most famous example of such a polynomial is certainly Euler's polynomial $f_{41}(x)=x^{2}+x+41$, which is prime for $0 \leq x \leq 39$. To date, no one has found a polynomial of the form $f_{A}(x)$ that represents distinct primes for more than the first 40 values of $x$. However, several people including Beeger [3, Lehmer 14], and Fung and Williams [7] have found polynomials which have higher asymptotic densities of prime values. According to Conjecture F, the function $C(\Delta)$ should provide a good indication of likely candidate polynomials. For example, the largest value of $C(\Delta)$ currently known [11] before this work is

$$
C(-13598858514212472187)=5.3670819 .
$$

The corresponding polynomial $x^{2}+x+3399714628553118047$ starts off slower than Euler's polynomial (only 24 primes for $x \leq 100$ compared to 87), but for $x \leq 10^{7}$ it assumes 2517022 prime values as compared to only 2208197 by Euler's polynomial. Notice that for Euler's polynomial we have $C(-163)=3.3197732$, so by Conjecture $\mathrm{F}$ we expect that it will assume fewer prime values asymptotically than $x^{2}+x+3399714628553118047$.

The purpose of this paper is to describe a new method for accurately computing $C(\Delta)$ for values of $|\Delta|$ up to 70 digits (under the Extended Riemann Hypothesis - ERH) and to provide some new values of $\Delta$ for which $C(\Delta)$ is larger than any value previously computed.

\section{Estimating $C(\Delta)$}

The infinite product representation (1.1) of $C(\Delta)$ converges very slowly; consequently, we need another method to approximate it more rapidly. We used the 
formula of Fung and Williams [7, which can be derived from (14) and (20) of Shanks [23]. For $\Delta<-4$ they show that

$$
C(\Delta)=\frac{c \pi^{3} \sqrt{|\Delta|}}{90 h_{\Delta}} \cdot \frac{1}{L\left(2, \chi_{\Delta}\right)} \prod_{\substack{p \mid \Delta \\ p \text { odd }}}\left(1-\frac{1}{p^{4}}\right) \prod_{q \geq 3}\left(1-\frac{2}{q(q-1)^{2}}\right),
$$

where $q$ denotes a prime such that $\left(\frac{\Delta}{q}\right)=1, h_{\Delta}$ is the ideal class number of the imaginary quadratic order $\mathcal{O}_{\Delta}$ and

$$
c= \begin{cases}\frac{5}{2} & \text { if } \Delta \equiv 1 \quad(\bmod 8) \\ \frac{1}{2} & \text { if } \Delta \equiv 5 \quad(\bmod 8) \\ \frac{15}{16} & \text { otherwise }\end{cases}
$$

Similarly, one can derive for $\Delta>0[11]$

$$
C(\Delta)=\frac{c \pi^{4} \sqrt{\Delta}}{180 R_{\Delta} h_{\Delta}} \cdot \frac{1}{L\left(2, \chi_{\Delta}\right)} \prod_{\substack{p \mid \Delta \\ p \text { odd }}}\left(1-\frac{1}{p^{4}}\right) \prod_{q \geq 3}\left(1-\frac{2}{q(q-1)^{2}}\right),
$$

where as above $h_{\Delta}$ is the class number of the real quadratic order $\mathcal{O}_{\Delta}$ and $R_{\Delta}$ is the regulator, i.e., the natural logarithm of the fundamental unit of $\mathcal{O}_{\Delta}$. Thus, in order to approximate $C(\Delta)$ we have to compute the class number (and regulator for $\Delta>0$ ) of the quadratic order $\mathcal{O}_{\Delta}$, factor $\Delta$, and estimate the infinite product

$$
P=\frac{1}{L\left(2, \chi_{\Delta}\right)} \cdot \prod_{q \geq 3}\left(1-\frac{2}{q(q-1)^{2}}\right) .
$$

Also, we note that because

$$
-\log \prod_{\substack{p \backslash \Delta \\ p \geq A}}\left(1-p^{-4}\right) \sim \sum_{\substack{p \backslash \Delta \\ p \geq A}} p^{-4}=O\left(A^{-4} \log _{A}|\Delta|\right),
$$

it is a simple matter to estimate the value of $\prod_{p \mid \Delta}\left(1-p^{-4}\right)$ very accurately without having to completely factor $\Delta$. Fortunately, $P$ converges much more quickly than (1.1), and, while we could use the method of [7] to estimate it rapidly, we found that a slight modification of the method of Bach [2] to evaluate $L\left(2, \chi_{\Delta}\right)$ produced an even faster technique for doing this. We will now briefly sketch this procedure. The notation, unless otherwise stated, is that of [2].

We first let $\chi$ be any non-principal character modulo $m$, and we put

$$
\begin{gathered}
B(x, \chi)=\prod_{p<x} \frac{p^{2}}{p^{2}-\chi(p)}, \quad \bar{B}(x, \chi)=\prod_{p \geq x} \frac{p^{2}}{p^{2}-\chi(p)} \\
F(x, \chi)=\prod_{q<x}\left(1-\frac{2}{q(q-1)^{2}}\right), \quad \bar{F}(x, \chi)=\prod_{q \geq x}\left(1-\frac{2}{q(q-1)^{2}}\right),
\end{gathered}
$$

where, as above, the values of $p$ are prime integers and the values of $q$ are odd primes such that $\left(\frac{\Delta}{q}\right)=1$. It is easy to deduce that

$$
|\log \bar{F}(x, \chi)| \leq 3 \sum_{n \geq x} \frac{1}{(n-1)^{3}}<\frac{2}{x^{2}} \quad(x>15) .
$$


By the reasoning in 2, we have

$$
\log \bar{B}(x, \chi)=\int_{x^{-}}^{\infty} \frac{d \Psi(t, \chi)}{t^{2} \log t} d t-T(x, \chi),
$$

where

$$
T(x, \chi)=\sum_{\substack{p^{k} \geq x \\ p<x}} \frac{\chi\left(p^{k}\right)}{k p^{2 k}} .
$$

The method of the proof of Lemma 5.1 of [2] can be used to establish that

$$
|T(x, \chi)| \leq 4 C\left[\frac{2}{3 x^{3 / 2} \log x}+\frac{3}{5 x^{5 / 3} \log 2}\right],
$$

where $C=1.25506$. Also, if

$$
\Psi^{1}(x, \chi)=\int_{0}^{x} \Psi(t, \chi) d t
$$

then under the ERH (Lemma 9.3 of [2]) we know that

$$
\left|\Psi^{1}(x, \chi)\right| \leq c(m) x^{3 / 2}+h(x)
$$

where

$$
c(m)=2 / 3(\log m+5 / 3)
$$

and

$$
h(x)=x \log x+(2 c(m)+1) x+3 c(m)+1 .
$$

If we integrate by parts twice, we get, on the assumption that $x$ is integral,

$$
\begin{aligned}
\int_{x^{-}}^{\infty} \frac{d \Psi(t, \chi)}{t^{2} \log t} d t=-\frac{\Psi(x-1, \chi)}{x^{2} \log x} & -\frac{\Psi^{1}(x, \chi)(2 \log x+1)}{x^{3} \log ^{2} x} \\
& +\int_{x}^{\infty} \Psi^{1}(t, \chi)\left(\frac{6 \log ^{2} t+5 \log t+2}{t^{4} \log ^{3} t}\right) d t
\end{aligned}
$$

We next define, for a given positive integer $x$,

$$
a_{i}=\frac{(x+i)^{2} \log (x+i)}{S(x)},
$$

where

$$
S(x)=\sum_{i=0}^{x-1}(x+i)^{2} \log (x+i) .
$$

Clearly

$$
\sum_{i=1}^{x-1} a_{i}=1
$$

We now consider

$$
E(x, \chi)=\sum_{i=0}^{x-1} a_{i} \log \bar{B}(x+i, \chi)
$$


NEW QUADRATIC POLYNOMIALS WITH HIGH DENSITIES OF PRIME VALUES 503

and note that

$$
\sum_{i=0}^{x-1} a_{i} \log B(x+i, \chi)+E(x, \chi)=\log L(2, \chi) .
$$

It follows that

$$
\left|\log L(2, \chi)-\sum_{i=0}^{x-1} a_{i} \log B(x+i, \chi)\right| \leq|E(x, \chi)|
$$

We put

$$
C^{*}(Q, \Delta)=w \sqrt{|\Delta|} \prod_{p \mid \Delta}\left(1-\frac{1}{p^{4}}\right) F\left(Q, \chi_{\Delta}\right) \exp \left\{-\sum_{i=0}^{Q-1} a_{i} \log B\left(Q+i, \chi_{\Delta}\right)\right\},
$$

where $\chi_{\Delta}$ is the Kronecker symbol $(\Delta / \cdot)$ and

$$
w= \begin{cases}c \pi^{3} /\left(90 h_{\Delta}\right) & \text { if } \Delta<0, \\ c \pi^{4} /\left(180 R_{\Delta} h_{\Delta}\right) & \text { if } \Delta>0 .\end{cases}
$$

We now note that if $k=5 \cdot 10^{-r}$, then

$$
\left|\frac{C(\Delta)-C^{*}(Q, \Delta)}{C(\Delta)}\right|<k
$$

when

$$
\left|\log C(\Delta)-\log C^{*}(Q, \Delta)\right|<\log (1+k),
$$

and $C^{*}(Q, \Delta)$ will approximate $C(\Delta)$ to $r$ figures of accuracy. By (2.8)

$$
\log C(\Delta)-\log C^{*}(Q, \Delta)=\log \bar{F}\left(Q, \chi_{\Delta}\right)-E\left(Q, \chi_{\Delta}\right) .
$$

Hence, by 2.1),

$$
\left|\log C(\Delta)-\log C^{*}(Q, \Delta)\right| \leq\left|E\left(Q, \chi_{\Delta}\right)\right|+\frac{2}{Q^{2}} \quad(Q>15) .
$$

Thus, we need to be able to bound $E\left(x, \chi_{\Delta}\right)$ in order to find a value for $Q$ such that (2.10) holds.

We note that by (2.2), (2.5), and (2.7), we get

$$
\begin{aligned}
|E(x, \chi)| \leq & \left|\sum_{i=0}^{x-1} a_{i} \frac{\Psi(x+i-1, \chi)}{(x+i)^{2} \log (x+i)}\right|+\left|\sum_{i=0}^{x-1} a_{i} \frac{\Psi^{1}(x+i, \chi)(2 \log (x+i)+1)}{(x+i)^{3} \log ^{2}(x+i)}\right| \\
& +\left|\sum_{i=0}^{x-1} a_{i} \int_{x+i}^{\infty} \Psi^{1}(t, \chi) \frac{6 \log ^{2} t+5 \log t+2}{t^{4} \log ^{3} t} d t\right|+\left|\sum_{i=0}^{x-1} a_{i} T(x+i, \chi)\right| .
\end{aligned}
$$

It is easy to see that

$$
\begin{aligned}
S(x) & >U(x):=\int_{0}^{x-1}(t+x)^{2} \log (t+x) d t \\
& =1 / 3\left[(2 x-1)^{3}(\log (2 x-1)-1 / 3)-x^{3}(\log x-1 / 3)\right] \\
& >2 x^{3} \log x
\end{aligned}
$$


when $x>3000$. Also,

$$
\sum_{i=0}^{x-1}(x+i)^{1 / 2}<\int_{0}^{x}(x+t)^{1 / 2} d t=\lambda x^{3 / 2}
$$

where $\lambda=2 / 3\left(2^{3 / 2}-1\right) \approx 1.2189514$. With these observations, (2.4), and (2.6) we get

$$
\begin{aligned}
\left|\sum_{i=0}^{x-1} a_{i} \frac{\Psi(x+i-1, \chi)}{(x+i)^{2} \log (x+i)}\right|=\frac{1}{S(x)} \mid & \left|\sum_{i=0}^{x-1} \Psi(x+i-1, \chi)\right| \\
& <\frac{\left(1+2^{3 / 2}\right) c(m) x^{3 / 2}}{U(x)}+\frac{h(x)+h(2 x)}{2 x^{3} \log x}
\end{aligned}
$$

and

$$
\begin{array}{r}
\left|\sum_{i=0}^{x-1} a_{i} \frac{\Psi^{1}(x+i, \chi)(2 \log (x+i)+1)}{(x+i)^{3} \log ^{2}(x+i)}\right| \leq \sum_{i=0}^{x-1} a_{i} \frac{c(m)(x+i)^{3 / 2}(2 \log (x+i)+1)}{(x+i)^{3} \log ^{2}(x+i)} \\
+\frac{h(x)(2 \log x+1)}{2 x^{3} \log x}
\end{array}
$$

because $h(x)(2 \log x+1) /\left(2 x^{3} \log x\right)$ is a decreasing function of $x$. It follows from (2.11), (2.12) and the definition of $a_{i}$ that

$$
\begin{aligned}
\left|\sum_{i=0}^{x-1} a_{i} \frac{\Psi^{1}(x+i, \chi)(2 \log (x+i)+1)}{(x+i)^{3} \log ^{2}(x+i)}\right| \leq \frac{c(m)}{U(x)}(2+ & \left.\frac{1}{\log x}\right) \lambda x^{3 / 2} \\
& +\frac{h(x)(2 \log x+1)}{2 x^{3} \log x} .
\end{aligned}
$$

It is also easy to deduce from (2.3), (2.6), (2.11), and (2.12) that

$$
\left|\sum_{i=0}^{x-1} a_{i} T(x+i, \chi)\right| \leq \frac{8 C \lambda x^{3 / 2}}{3 S(x)}+\frac{12 C}{(5 \log 2) x^{5 / 3}} .
$$

We note that by (2.4)

$$
\begin{aligned}
& \left|\sum_{i=0}^{x-1} a_{i} \int_{x+i}^{\infty} \Psi^{1}(t, \chi) \frac{6 \log ^{2} t+5 \log t+2}{t^{4} \log ^{3} t} d t\right| \\
& \leq c(m) \sum_{i=0}^{x-1} a_{i}\left(\frac{6}{\log (x+i)}+\frac{5}{\log ^{2}(x+i)}+\frac{2}{\log ^{3}(x+i)}\right) \int_{x+i}^{\infty} t^{-5 / 2} d t \\
& \quad+\int_{x}^{\infty} \frac{h(x)}{t^{4}}\left(\frac{6}{\log t}+\frac{5}{\log ^{2} t}+\frac{2}{\log ^{3} t}\right) d t .
\end{aligned}
$$

Also, by (2.12) we have

$$
\begin{aligned}
\sum_{i=0}^{x-1} a_{i}\left(\frac{6}{\log (x+i)}+\frac{5}{\log ^{2}(x+i)}+\frac{2}{\log ^{3}(x+i)}\right) & (x+i)^{-3 / 2} \\
\leq & \frac{\lambda x^{3 / 2}}{S(x)}\left(6+\frac{5}{\log x}+\frac{2}{\log ^{2} x}\right) .
\end{aligned}
$$


TABlE 2.1. $Q$ values for approximating $C(\Delta)$.

\begin{tabular}{|c|c|}
\hline $\log _{10}|\Delta|$ & $Q$ \\
\hline \hline 30 & 525500 \\
35 & 576000 \\
40 & 624500 \\
45 & 670500 \\
50 & 715000 \\
55 & 758000 \\
60 & 799500 \\
65 & 839500 \\
70 & 879000 \\
75 & 917000 \\
\hline
\end{tabular}

If, after Bach, we define the linear functional $T_{x}$ on any function $f$ which is positive and non-decreasing but grows sufficiently slowly that $f(x)(1+2 \log x) /\left(x^{3} \log x\right)$ is decreasing, as

$$
\begin{aligned}
T_{x}(f)=\frac{f(x)+f(2 x)}{2 x^{3} \log x}+\frac{f(x)(2 \log x+1)}{2 x^{3} \log x} & \\
& +\int_{x}^{\infty} \frac{f(t)}{t^{4}}\left(\frac{6}{\log t}+\frac{5}{\log ^{2} t}+\frac{2}{\log ^{3} t}\right) d t,
\end{aligned}
$$

we see by (2.13), (2.14), (2.15) and our results above that

$$
\begin{aligned}
|E(x, \chi)| \leq \frac{c(m) x^{3 / 2}}{U(x)}\left(1+2^{3 / 2}+\right. & 6 \lambda)+\frac{13 c(m) \lambda}{6 x^{3 / 2} \log ^{2} x}+\frac{2 \lambda c(m)}{3 x^{3 / 2} \log ^{3} x} \\
& +\frac{4 C \lambda}{3 x^{3 / 2} \log x}+\frac{12 C}{(5 \log 2) x^{5 / 3}}+T_{x}(h) .
\end{aligned}
$$

Since (for $\alpha<3$ )

$$
\begin{gathered}
T_{x}\left(x^{\alpha}\right) \leq \frac{1}{x^{3-\alpha} \log x}\left[\left(3+2^{\alpha}+\frac{6}{3-\alpha}\right)+\left(1+\frac{5}{3-\alpha}\right) \frac{1}{\log x}+\frac{2}{3-\alpha} \frac{1}{(\log x)^{2}}\right], \\
T_{x}(x \log x) \leq \frac{1}{x^{2}}\left[8+\frac{2 \log 2+6}{\log x}+\frac{1}{\log ^{2} x}\right],
\end{gathered}
$$

it is easy to use (2.16) to find the least value of $Q$ such that

$$
\left|E\left(Q, \chi_{\Delta}\right)\right|+2 / Q^{2}<\log (1+k) .
$$

Since the dominant term of (2.16) is $O\left(x^{-3 / 2} \log m\right)$, we would expect

$$
Q=O\left(10^{2 r / 3}(\log |\Delta|)^{2 / 3}\right) .
$$

Of course, since the bound on $E\left(Q, \chi_{\Delta}\right)$ and (later) the correctness of $h_{\Delta}$ and $R_{\Delta}$ are all conditional on the truth of the ERH, our approximation of $C(\Delta)$ is as well. In Table 2.1 we list the $Q$ values required to approximate $C(\Delta)$ to 8 significant figures for various sizes of $\Delta$. Naturally, any $Q$ which works for a given size of $\Delta$ also works for all smaller values of $\Delta$. We note here that since $\Delta \equiv 1(\bmod 4)$ and our values of $\Delta$ will be squarefree in the sequel, we may use $m=|\Delta|$. These same properties of $\Delta$ are assumed in Table 2.1 . 


\section{Computing the Class Number AND REgulator}

The majority of the computation time spent in using (2.9) to approximate $C(\Delta)$ is in computing the class number and regulator of the quadratic order $\mathcal{O}_{\Delta}$. We used the method described in [12] (Algorithm 4.3). The underlying strategy of this algorithm is the same as that of Hafner and McCurley [9] and its variants [6], [4], [1], 5]. Suppose we have computed a factor base $F B=\left\{\mathfrak{p}_{1}, \ldots, \mathfrak{p}_{k}\right\}$ consisting of invertible prime ideals such that the equivalence classes of some subset of $F B$ generates the class group $C l_{\Delta}$ of $\mathcal{O}_{\Delta}$. For $\vec{v} \in \mathbb{Z}^{k}$ we define

$$
F B^{\vec{v}}=\prod_{i=1}^{k} \mathfrak{p}_{i}^{v_{i}},
$$

where $\mathfrak{p}_{i} \in F B$. We call $\vec{v}$ a relation if the ideal $F B^{\vec{v}}$ is principal, i.e., $F B^{\vec{v}} \sim \mathcal{O}_{\Delta}$. The algorithm then produces a generating system $L=\left\{\vec{v}_{1}, \ldots, \vec{v}_{n}\right\}$ of the relation lattice

$$
\Lambda=\left\{\vec{v} \in \mathbb{Z}^{k} \mid F B^{\vec{v}} \sim \mathcal{O}_{\Delta}\right\},
$$

which is the kernel of the homomorphism

$$
\mathbb{Z}^{k} \rightarrow C l_{\Delta}, \quad \vec{v} \rightarrow F B^{\vec{v}}
$$

Since the equivalence classes of the ideals of $F B$ generate the class group, it follows that the homomorphism (3.2) is surjective, and we have

$$
C l_{\Delta} \cong \mathbb{Z}^{k} / \Lambda \text {. }
$$

This implies that $\Lambda$ is a $k$-dimensional lattice and its determinant is equal to $h_{\Delta}$. Also, the relation matrix $A=\left(\vec{v}_{1}^{T}, \ldots, \vec{v}_{n}^{T}\right)$, the matrix formed by taking the relations $\vec{v}_{i}$ as columns, has rank $k$. The diagonal elements which are greater than 1 in $S$, the Smith normal form of $A$, are precisely the elementary divisors of $C l_{\Delta}$. Thus, in addition to $h_{\Delta}$, we get the structure of $C l_{\Delta}$ as a direct product of cyclic subgroups with very little extra effort.

This strategy can easily be extended to compute class groups and regulators of real quadratic orders [4, 1]. In this case, we compute relations of the form $(\vec{v}, \log |\gamma|)$, where $F B^{\vec{v}}=(\gamma)$, i.e., $\gamma$ generates the principal ideal $F B^{\vec{v}}$. We produce a generating system

$$
L^{\prime}=\left\{\left(\vec{v}_{1}, \log \left|\gamma_{1}\right|\right),\left(\vec{v}_{2}, \log \left|\gamma_{2}\right|\right), \ldots,\left(\vec{v}_{l}, \log \left|\gamma_{l}\right|\right)\right\}
$$

of the extended relation lattice

$$
\Lambda^{\prime}=\left\{(\vec{v}, \log |\gamma|) \in \mathbb{Z}^{k} \times \mathbb{R} \mid F B^{\vec{v}}=(\gamma)\right\} .
$$

Then, if $\Lambda$ is the part of $\Lambda^{\prime}$ in $\mathbb{Z}^{k}$, as before we have $C l_{\Delta} \cong \mathbb{Z}^{k} / \Lambda$. Furthermore, it can be shown [4] that $\operatorname{det}\left(\Lambda^{\prime}\right)=h_{\Delta} R_{\Delta}$, so by computing this determinant we also get the regulator.

The major difference between our approach and that of [9], 6], etc., is in the way the generating system of the relation lattice is produced. The solution employed by contemporary algorithms is to attempt to factor randomly produced ideals over the factor base. We replace this step by a sieve-based strategy similar to that used in the MPQS factoring algorithm [24]. The idea of employing sieving to compute relations in similar contexts was first suggested by Seysen [22], and later by Paulus [20]. 
In the MPQS, one sieves over quadratic polynomials $F(x)=a x^{2}+b x+c$ in order to find certain values of $x$ for which $F(x)$ completely factors over a finite factor base of prime integers. By sieving a polynomial $F(x)$ over an interval, we mean testing each value of $X$ in a given interval as to whether all the prime factors of $F(X)$ are contained in a finite, given set. The observation that $F(X) \equiv F(X+i p)$ $(\bmod p)$ for $i \in \mathbb{Z}, p$ prime, allows one to use a sieve to perform this test rather than evaluating every value of $F(x)$ and attempting to factor it.

In our case, we first compute an ideal $\mathfrak{a}$ as a power-product of the prime ideals in our factor base $F B$, i.e., $\mathfrak{a}=F B^{\vec{e}}$ for some $\vec{e} \in \mathbb{Z}^{k}$. The vector $\vec{e}$ is sparse with non-zero entries \pm 1 . In [12, we provide further details on how $\vec{e}$ is selected. Then, we search for integers $X$ and $Y$ such that $f(X, Y)=a X^{2}+b X Y+c Y^{2}$, the norm form of $\mathfrak{a}$, factors over the norms of the ideals in $F B$. For each such pair $(X, Y)$, there exists a quadratic number $\gamma$ such that $\mathfrak{a} /(\gamma)=\mathfrak{b}^{-1}$ splits over the factor base. As shown in [12, we can explicitly compute $\mathfrak{b}$ and its decomposition over $F B$ easily. Since $\mathfrak{a}$ splits over $F B$ by construction, we have that $\mathfrak{a} \mathfrak{b}=(\gamma)$ yields a relation.

The main work in generating relations with the strategy outlined above is finding smooth values of the quadratic polynomial $f(x, y)$. It is certainly possible to sieve $f(x, y)$ in two dimensions. However, most sieve-based factoring algorithms, including the MPQS, work exclusively with univariate quadratic polynomials. Hence, in order to parallel these factoring methods as closely as possible, we also work with the univariate polynomials $F(x)=f(x, 1)=a x^{2}+b x+c$.

Thus, the problem of finding relations for class group computation is reduced to the same problem as finding relations in the MPQS factoring algorithm. A large amount of effort has been invested in making the MPQS and its variants as efficient as possible, and we make use of as many of these techniques as possible, most notably self-initialization. The use of these sieving methods results in a dramatic increase in performance. See [12] for more details and computational results.

\section{Previous Results}

The example $\Delta=-13598858514212472187$ and others like it were generated using the MSSU [18, 17, a numerical sieving device capable of searching for solutions to sets of simultaneous linear congruences at the rate of over $4 \times 10^{12}$ candidates per second. A typical problem solvable by such sieving devices is as follows. A set of moduli is first specified, and then a set of acceptable residues is chosen for each modulus. The sieve then searches for integer solutions $x>L$ for some lower bound $L$ such that $x$ is congruent to any one of the acceptable residues modulo each of the corresponding moduli.

The strategy employed to find values of $\Delta$ with large $C(\Delta)$ values was to search for values of $\Delta \equiv 5(\bmod 8)$ for which $\left(\frac{\Delta}{q}\right)=-1$ for all odd primes $q$ less than or equal to some bound $p$. Clearly this has the effect of maximizing the leading terms in the infinite product representation of $C(\Delta)$. The problem of finding such values of $\Delta$ can be formulated as a sieve problem by fixing as moduli 8 and the primes less than or equal to $p$. For each of these primes $q$ the acceptable residues are the integers $x$ such that $1 \leq x<q$ and $x$ is a quadratic non-residue modulo $q$. The acceptable residue for 8 is 5 , since we want solutions $\Delta \equiv 5(\bmod 8)$. The sieve then searches for integers which are congruent to one of the acceptable residues for each modulus. 
Following Lehmer [14, we define the symbol $N_{p}$ to represent the least positive integer congruent to 3 modulo 8 such that $\left(\frac{-N_{p}}{q}\right)=-1$ for all odd primes $q \leq p$. Lehmer computed the first table of $N_{p}$ values for $p \leq 107$. Lehmer, Lehmer, and Shanks extended these computations in 15, Problem III, to values of $p \leq 163$, and Lehmer also computed the next three values up to $p=181$, but did not publish them. In [1], the MSSU was used to extend these computations further, and values of $N_{p}$ up to $p=277$ and least prime solutions of $N_{p}$ up to $p=269$ were found. Tables 4.1 and 4.2 are reproduced from [11], and contain all the currently known values of $N_{p}$ and the least prime solutions of $N_{p}$, respectively.

TABle 4.1. $N_{p}$ - Least Solutions

\begin{tabular}{|r|r|r|l|}
\hline \multicolumn{1}{|c|}{$N_{p}$} & \multicolumn{1}{|c|}{$h_{-N_{p}}$} & $C\left(-N_{p}\right)$ \\
\hline \hline 3 & 19 & 1 & 0.94222046 \\
5,7 & 43 & 1 & 1.6297209 \\
11,13 & 67 & 1 & 2.0873308 \\
$17, \ldots, 37$ & 163 & 1 & 3.3197732 \\
41,43 & 77683 & 22 & 3.3003388 \\
47 & 1333963 & 79 & 3.8123997 \\
53,59 & 2404147 & 107 & 3.7793704 \\
61 & 20950603 & 311 & 3.8410195 \\
67 & 36254563 & 432 & 3.6365197 \\
71 & 51599563 & 487 & 3.8514289 \\
73,79 & 96295483 & 665 & 3.8528890 \\
83 & 114148483 & 692 & 4.0332358 \\
$89, \ldots, 103$ & 269497867 & 1044 & 4.1092157 \\
107 & 585811843 & 1536 & 4.1185705 \\
109,113 & 52947440683 & 13909 & 4.3245257 \\
127 & 71837718283 & 15204 & 4.6097143 \\
131,137 & 229565917267 & 29351 & 4.2679170 \\
139 & 575528148427 & 44332 & 4.4746374 \\
$149, \ldots, 163$ & 1432817816347 & 70877 & 4.4163429 \\
167 & 6778817202523 & 149460 & 4.5565681 \\
173 & 16501779755323 & 223574 & 4.7524812 \\
179,181 & 30059924764123 & 296475 & 4.8379057 \\
$191,193,197$ & 110587910656507 & 553436 & 4.9711959 \\
199 & 4311527414591923 & 3791896 & 4.5293043 \\
211,223 & 10472407114788067 & 5798780 & 4.6162389 \\
$227, \ldots, 241$ & 22261805373620443 & 8035685 & 4.8576312 \\
251 & 132958087830686827 & 19412108 & 4.9146545 \\
257 & 441899002218793387 & 33684408 & 5.1635913 \\
263,269 & 2278509757859388307 & 77949544 & 5.0669199 \\
271 & 5694230275645018963 & 119705436 & 5.2163043 \\
277 & 9828323860172600203 & 156104956 & 5.2552050 \\
\hline & & & \\
\hline
\end{tabular}


Table 4.2. $N_{p}$ - Least Prime Solutions

\begin{tabular}{|r|r|r|l|}
\hline \multicolumn{1}{|c|}{$N_{p}$} & $h_{-N_{p}}$ & \multicolumn{1}{|c|}{$C\left(-N_{p}\right)$} \\
\hline \hline 3 & 19 & 1 & 0.94222046 \\
5,7 & 43 & 1 & 1.6297209 \\
11,13 & 67 & 1 & 2.0873308 \\
$17, \ldots, 37$ & 163 & 1 & 3.3197732 \\
41 & 222643 & 33 & 3.7289570 \\
43,47 & 1333963 & 79 & 3.8123997 \\
53,59 & 2404147 & 107 & 3.7793704 \\
61 & 20950603 & 311 & 3.8410195 \\
67,71 & 51599563 & 487 & 3.8514289 \\
73,79 & 96295483 & 665 & 3.8528890 \\
83 & 146161723 & 857 & 3.6832906 \\
89 & 1408126003 & 2293 & 4.2771747 \\
$97,101,103$ & 3341091163 & 3523 & 4.2878711 \\
$107,109,113$ & 52947440683 & 13909 & 4.3245257 \\
127 & 193310265163 & 26713 & 4.3024065 \\
131,137 & 229565917267 & 29351 & 4.2679170 \\
139 & 915809911867 & 59801 & 4.1834705 \\
$149, \ldots, 163$ & 1432817816347 & 70877 & 4.4163429 \\
$167, \ldots, 181$ & 30059924764123 & 296475 & 4.8379057 \\
191 & 3126717241727227 & 3201195 & 4.5685162 \\
$193,197,199$ & 8842819893041227 & 5188215 & 4.7414735 \\
211,223 & 13688678408873323 & 6524653 & 4.6907580 \\
$227, \ldots, 241$ & 22261805373620443 & 8035685 & 4.8576312 \\
251 & 4908856524312968467 & 121139393 & 4.7847955 \\
$257,263,269$ & 7961860547428719787 & 140879803 & 5.2409110 \\
\hline
\end{tabular}

Similarly, we define the symbol $M_{p}$ to represent the least positive integer congruent to 5 modulo 8 such that $\left(\frac{M_{p}}{q}\right)=-1$ for all odd primes $q \leq p$. We would expect, due to Conjecture $\mathrm{F}$, that $\left|f_{A}(x)\right|$ will have a large density of prime values when $A=\left(1-M_{p}\right) / 4$. According to Poletti [21], Beeger was the first to make a table of $M_{p}$ values; he listed them up to $p=59$. Lehmer, Lehmer, and Shanks [15], Problem VI, extended this table in 1970 up to $p=139$, and Lehmer produced one more value for $p=163$, but did not publish it. The MSSU was used to extend the table further, to $p=283$ and $p=263$ for least prime solutions. Tables 4.3 and 4.4 again reproduced from [11], contain all the currently known values of $M_{p}$ and the least prime solutions of $M_{p}$, respectively.

TABLE 4.3. $M_{p}$ - Least Solutions

\begin{tabular}{|c|c|c|c|c|}
\hline$p$ & $M_{p}$ & $R_{M_{p}}$ & $h_{M_{p}}$ & $C\left(M_{p}\right)$ \\
\hline 3 & 5 & 0.4812 & 1 & 1.7733051 \\
\hline 5 & 53 & 1.9657 & 1 & 1.3831458 \\
\hline 7,11 & 173 & 2.5708 & 1 & 2.0427655 \\
\hline 13 & 293 & 2.8366 & 1 & 2.4386997 \\
\hline 17 & 437 & 3.0422 & 1 & 2.7933935 \\
\hline
\end{tabular}


TABLE 4.3. $M_{p}$ - Least Solutions (continued)

\begin{tabular}{|r|r|r|c|c|}
\hline \multicolumn{1}{|c|}{$M_{p}$} & $R_{M_{p}}$ & $h_{M_{p}}$ & $C\left(M_{p}\right)$ \\
\hline \hline 19,23 & 9173 & 12.4722 & 1 & 3.1227858 \\
29 & 24653 & 5.0562 & 4 & 3.1631443 \\
$31,37,41$ & 74093 & 7.2159 & 5 & 3.0809338 \\
43 & 170957 & 16.9391 & 3 & 3.3299831 \\
$47,53,59$ & 214037 & 28.9536 & 2 & 3.2704656 \\
61 & 2004917 & 48.2972 & 3 & 4.0077796 \\
67 & 44401013 & 352.5078 & 2 & 3.8743032 \\
71 & 71148173 & 140.5395 & 6 & 4.1026493 \\
73,79 & 154554077 & 694.9131 & 2 & 3.6684052 \\
$83,89,97$ & 163520117 & 152.1367 & 9 & 3.8307572 \\
101,103 & 261153653 & 512.3272 & 3 & 4.3158954 \\
$107,109,113$ & 1728061733 & 4021.1400 & 1 & 4.2447622 \\
127 & 9447241877 & 1252.3775 & 7 & 4.5541813 \\
131 & 19553206613 & 6209.5055 & 2 & 4.6250203 \\
137,139 & 49107823133 & 18804.6808 & 1 & 4.8420287 \\
$149, \ldots, 163$ & 385995595277 & 27068.0628 & 2 & 4.7144914 \\
167 & 13213747959653 & 330785.2663 & 1 & 4.5147795 \\
173 & 14506773263237 & 331149.0061 & 1 & 4.7257867 \\
179,181 & 57824199003317 & 165998.4596 & 4 & 4.7059530 \\
191,193 & 160909740894437 & 275610.2629 & 4 & 4.7279560 \\
197,199 & 370095509388197 & 794079.6472 & 2 & 4.9779329 \\
211 & 1409029796180597 & 3130386.6897 & 1 & 4.9274990 \\
223 & 4075316253649373 & 5291574.7242 & 1 & 4.9577054 \\
$227,229,233$ & 18974003020179917 & 2737025.3979 & 4 & 5.1711431 \\
239,241 & 224117990614052477 & 10257518.4583 & 4 & 4.7415726 \\
$251,257,263$ & 637754768063384837 & 22908547.7970 & 3 & 4.7753226 \\
$269, \ldots .283$ & 4472988326827347533 & 14462868.4419 & 12 & 5.0085747 \\
\hline
\end{tabular}

TABle 4.4. $M_{p}$ - Least Prime Solutions

\begin{tabular}{|r|r|r|c|c|}
\hline \multicolumn{1}{|c|}{$M_{p}$} & $R_{M_{p}}$ & $h_{M_{p}}$ & $C\left(M_{p}\right)$ \\
\hline \hline 3 & 5 & 0.48121 & 1 & 1.7733051 \\
5 & 53 & 1.96572 & 1 & 1.3831458 \\
7,11 & 173 & 2.57081 & 1 & 2.0427655 \\
13 & 293 & 2.83665 & 1 & 2.4386997 \\
17 & 2477 & 6.47234 & 1 & 3.1173079 \\
19,23 & 9173 & 12.47223 & 1 & 3.1227858 \\
29 & 61613 & 36.23370 & 1 & 2.7929099 \\
$31,37,41$ & 74093 & 7.21597 & 5 & 3.0809338 \\
43 & 170957 & 16.93918 & 3 & 3.3299831 \\
47 & 360293 & 68.23691 & 1 & 3.6032397 \\
53 & 679733 & 92.04349 & 1 & 3.6713558 \\
59,61 & 2004917 & 48.29722 & 3 & 4.0077796 \\
67 & 69009533 & 869.69643 & 1 & 3.9166092 \\
71 & 138473837 & 1369.29769 & 1 & 3.5221802 \\
\hline
\end{tabular}


TABLE 4.4. $M_{p}$ - Least Prime Solutions (continued)

\begin{tabular}{|r|r|r|c|c|}
\hline \multicolumn{1}{|c|}{$M_{p}$} & \multicolumn{1}{|c|}{$R_{M_{p}}$} & $h_{M_{p}}$ & $C\left(M_{p}\right)$ \\
\hline \hline 73 & 237536213 & 1725.64096 & 1 & 3.6624765 \\
79 & 384479933 & 2087.35754 & 1 & 3.8534093 \\
83 & 883597853 & 3018.26471 & 1 & 4.0411818 \\
$89, \ldots, 113$ & 1728061733 & 4021.14004 & 1 & 4.2447622 \\
127 & 9447241877 & 1252.37753 & 7 & 4.5541813 \\
$131,137,139$ & 49107823133 & 18804.68086 & 1 & 4.8420287 \\
149 & 1843103135837 & 119080.85359 & 1 & 4.6828076 \\
151,157 & 4316096218013 & 192239.83257 & 1 & 4.4390420 \\
163,167 & 15021875771117 & 344898.80858 & 1 & 4.6165765 \\
173,179 & 82409880589277 & 804942.51462 & 1 & 4.6336310 \\
181 & 326813126363093 & 1551603.41110 & 1 & 4.7874230 \\
191,193 & 390894884910197 & 1650908.48845 & 1 & 4.9214877 \\
197 & 1051212848890277 & 547589.04349 & 5 & 4.8659116 \\
$199,211,223$ & 4075316253649373 & 5291574.72421 & 1 & 4.9577054 \\
227 & 274457237558283317 & 45653225.95687 & 1 & 4.7155029 \\
229 & 443001676907312837 & 6097479.67224 & 9 & 4.9843291 \\
233 & 599423482887195557 & 65388978.22854 & 1 & 4.8658247 \\
239 & 614530964726833997 & 64783176.97206 & 1 & 4.9730080 \\
$241, \ldots, 263$ & 637754768063384837 & 22908547.79705 & 3 & 4.7753226 \\
\hline
\end{tabular}

The example $\Delta=-13598858514212472187$ was found by using the MSSU to generate all values of $\Delta$ such that $-2 \times 10^{19}<\Delta<10^{19}, \Delta \equiv 5(8)$, and $(\Delta / q)=-1$ for all odd primes $q \leq 199$. By restricting to the primes less than 200 more results were generated than if a larger bound had been used. Furthermore, the sieve runs faster if fewer moduli are used, and thus a larger number of candidates could be tested. For the several thousand numbers that resulted, $C(\Delta)$ was computed using the Shanks heuristic [19, p.283] to calculate the class numbers when $\Delta>0$, and the technique of the previous section when $\Delta<0$. The $C(\Delta)$-hichamps for the cases $\Delta<0$ and $\Delta>0$ were then selected, i.e., those $\Delta$ with the property that their corresponding $C(\Delta)$ value is greater than that of any $\Delta$ of smaller magnitude found by the sieve.

$C(\Delta)$ was evaluated correct to 8 figures for all of these $C(\Delta)$-hichamps by using the previously described technique with the class numbers and regulators computed as in [11. No deviations from the results given by the Shanks heuristic were found. Table 4.5 contains the $C(\Delta)$-hichamps for the negative values of $\Delta$ and Table 4.6 contains the $C(\Delta)$-hichamps for the positive values of $\Delta$. The largest $C(\Delta)$ value found by this method is the aforementioned $C(-13598858514212472187)=$ 5.3670819 .

By using Littlewood's bounds on $L\left(1, \chi_{\Delta}\right)$ [16], it is easy to see [18] that under the ERH

$$
C(\Delta)<\{1+o(1)\} e^{\gamma} \log \log |\Delta|,
$$

where $\gamma$ is Euler's constant. Indeed, if we define

$$
r(\Delta)=C(\Delta) / e^{\gamma} \log \log |\Delta|,
$$


TABLE 4.5. $C(\Delta)$-hichamps $(\Delta<0)$.

\begin{tabular}{|r|r|c|c|}
\hline \multicolumn{1}{|c|}{$\Delta$} & \multicolumn{1}{c|}{$h_{\Delta}$} & $r(\Delta)$ & $C(\Delta)$ \\
\hline \hline-4311527414591923 & 3791896 & 0.70964311 & 4.5293043 \\
-5513463660887323 & 4214276 & 0.72070730 & 4.6086597 \\
-8842819893041227 & 5188215 & 0.73881217 & 4.7414735 \\
-11779882219755787 & 5904498 & 0.74766420 & 4.8086435 \\
-14363876114143483 & 6478729 & 0.75133104 & 4.8393795 \\
-15326624594334307 & 6664840 & 0.75401443 & 4.8590033 \\
-30462609261723907 & 9340770 & 0.75475096 & 4.8883007 \\
-32779240456803163 & 9520419 & 0.76778682 & 4.9753684 \\
-50792117776428667 & 11782274 & 0.76982885 & 5.0043010 \\
-221328140358231307 & 24591656 & 0.76210545 & 5.0050646 \\
-234391954943494723 & 24980688 & 0.77179828 & 5.0706939 \\
-369885383792662483 & 31346105 & 0.77034080 & 5.0766794 \\
-441899002218793387 & 33684408 & 0.78260083 & 5.1635912 \\
-554395014308976163 & 37602038 & 0.78412438 & 5.1814176 \\
-803608018073876563 & 45224688 & 0.78297632 & 5.1864453 \\
-2038991582966171563 & 71351592 & 0.78588177 & 5.2369507 \\
-2039953459173530587 & 70825967 & 0.79181939 & 5.2765336 \\
-6849319464662435083 & 128288704 & 0.79508448 & 5.3384020 \\
-13598858514212472187 & 179800672 & 0.79604287 & 5.3670819 \\
\hline
\end{tabular}

TABLE 4.6. $C(\Delta)$-hichamps $(\Delta>0)$.

\begin{tabular}{|r|c|r|c|c|}
\hline \multicolumn{1}{|c|}{$\Delta$} & $h_{\Delta}$ & \multicolumn{1}{c|}{$R_{\Delta}$} & $r(\Delta)$ & $C(\Delta)$ \\
\hline \hline 370095509388197 & 2 & 794079.64725 & 0.79561686 & 4.9779328 \\
16710980998953317 & 2 & 5296924.24250 & 0.77763395 & 5.0144216 \\
18974003020179917 & 4 & 2737025.39798 & 0.80118713 & 5.1711431 \\
587108439330001613 & 2 & 30377994.30089 & 0.78408776 & 5.1831340 \\
2430946649400343037 & 4 & 30781378.01108 & 0.78019116 & 5.2048129 \\
3512773592849667053 & 1 & 146959147.17623 & 0.78399584 & 5.2422843 \\
4927390995446922917 & 2 & 86988957.82243 & 0.78257150 & 5.2437622 \\
\hline
\end{tabular}

then as $\Delta$ increases we would expect that extreme values of the $r(\Delta)$ would tend to approach 1 if the ERH is true. Indeed, it is possible to use a result of Joshi 13] to show unconditionally that for any given positive $\varepsilon<1$, there exists an infinitude of values of $\Delta$ such that $r(\Delta)>(1+\varepsilon) / 2$. Thus, the closeness of $r(\Delta)$ to 1 provides an indication of how good our $C(\Delta)$ values are in relation to the size of $\Delta$. We have listed the $r(\Delta)$ values corresponding to the $C(\Delta)$ values in both Table 4.5 and Table 4.6. The largest $r(\Delta)$ value we found was 0.80118713 , corresponding to $\Delta=18974003020179917$.

\section{Generating $\Delta$ with larger $C(\Delta)$ values}

The MSSU and other sieving devices only allow a fixed number of moduli to be used at one time. Furthermore, when many moduli are used solutions can be quite rare. Hence, we used an unpublished idea of Lehmer which he employed to find the 
TABle 5.1. $A_{p}^{-}$and $A_{p}^{+}$values.

\begin{tabular}{|c|r|r|}
\hline$p$ & \multicolumn{1}{|c|}{$A_{p}^{-}$} & \multicolumn{1}{c|}{$A_{p}^{+}$} \\
\hline \hline 257 & 93 & 43 \\
277 & 942 & 1457 \\
307 & 8138 & 1730 \\
331 & 75586 & 203909 \\
353 & 4893645 & 3261415 \\
\hline
\end{tabular}

20 digit value of $\Delta$ with small $L\left(1, \chi_{\Delta}\right)$ that appears in [15, p.439]. Lehmer's idea allows us to find solutions $\Delta$ with $\left(\frac{\Delta}{q}\right)=-1$ for all $q \leq p$ while using fewer moduli for the sieve than would otherwise be required. To this end, we examined negative discriminants of the form

$$
\Delta=-\left(A_{p}^{-}+B_{p} X\right)
$$

and positive discriminants of the form

$$
\Delta=A_{p}^{+}+B_{p} X
$$

where

$$
B_{p}=\prod_{\substack{q \geq 233 \\ q \text { prime }}}^{p} q
$$

and $\left(\frac{-A_{p}^{-}}{q}\right)=\left(\frac{A_{p}^{+}}{q}\right)=-1$ for all primes $q(233 \leq q \leq p)$. We used five different values of $p$ ranging from 257 to 353 , and the least non-square values of $A_{p}^{-}$and $A_{p}^{+}$for each $p \in\{257,277,307,331,353\}$, which are given in Table 5.1. Our values of $B_{p}$, $A_{p}^{-}$, and $A_{p}^{+}$were selected so that we could generate solutions with approximately $30,40,50,60$, and 70 decimal digits, respectively.

For the case $\Delta=-\left(A_{p}^{-}+B_{p} X\right)<0$, we ran five separate sieve jobs corresponding to each of the five pairs $\left(A_{p}^{-}, B_{p}\right), p \in\{257,277,307,331,353\}$. We employed the MSSU to sieve on values of $X>0$ using as moduli 8 and primes $q_{1}, q_{2}, \ldots, q_{m}$ with $q_{m} \leq 229$. For each $q_{i}$, the acceptable residues were the values of $x$ such that $0 \leq x<q_{i}$ and $\left(\frac{-\left(A_{p}^{-}+B_{p} x\right)}{q_{i}}\right)=-1$. The observation that if $\left(\frac{y}{q}\right)=-1$ then $x \equiv\left(y+A_{p}^{-}\right)\left(-B_{p}\right)^{-1}(\bmod q)$ satisfies $\left(\frac{-\left(A_{p}^{-}+B_{p} x\right)}{q}\right)=-1$ allows one to easily determine all the acceptable residues for any given modulus $q$. In order to ensure that $-\left(A_{p}^{-}+B_{p} X\right) \equiv 5(\bmod 8)$ we use $x \equiv\left(5+A_{p}^{-}\right)\left(-B_{p}\right)^{-1}(\bmod 8)$ as the single acceptable residue for the modulus 8 . Thus, each solution $X$ found by the sieve which is congruent to one of the acceptable residues modulo 8 and every odd prime $q_{i} \leq 229$ yields a value of $\Delta=-\left(A_{p}^{-}+B_{p} X\right)$ such that $\left(\frac{\Delta}{q}\right)=-1$ for all odd primes $q$ less than or equal to $257,277,307,331$, and 353 , for each of the five pairs $\left(A_{p}^{-}, B_{p}\right)$. Notice that in order to find these $\Delta$ values we need only sieve with primes less than or equal to 229 .

We ran another five sieve jobs for the cases $\Delta=\left(A_{p}^{+}+B_{p} X\right)>0$ corresponding to the five pairs $\left(A_{p}^{+}, B_{p}\right), p \in\{257,277,307,331,353\}$. Again, we used as moduli 8 and the odd primes $q_{i} \leq 229$. The single acceptable residue for 8 is $\left(5-A_{p}^{+}\right)\left(B_{p}\right)^{-1} \bmod$ 8 , and the residues for each odd modulus $q$ are given by $\left(y-A_{p}^{+}\right)\left(B_{p}\right)^{-1} \bmod q$ for each $0 \leq y<q$ such that $\left(\frac{y}{q}\right)=-1$. Similarly, we obtained solutions $\Delta=A_{p}^{+}+B_{p} X$ 
TABle 5.2. $X$ values yielding $\Delta=-\left(A_{p}^{-}+B_{p} X\right)$ with large $C(\Delta)$.

\begin{tabular}{|c||c|c||c|c|}
\hline \multicolumn{1}{|c||}{} & \multicolumn{2}{c||}{ Composite $\Delta$} & \multicolumn{2}{c|}{ Prime $\Delta$} \\
\hline$p$ & $X$ & $n_{p}$ & $X$ & $n_{p}$ \\
\hline \hline 257 & 466615859130369190 & 30 & 450663765848215486 & 30 \\
277 & 370813410258174265 & 40 & 216364723669119361 & 39 \\
307 & 47981058088400465 & 49 & 177458814519025865 & 49 \\
331 & 180678079710346857 & 59 & 395114060043264249 & 60 \\
353 & 628306272374561842 & 70 & 22664467457614162 & 69 \\
\hline
\end{tabular}

TABLE 5.3. $X$ values yielding $\Delta=A_{p}^{+}+B_{p} X$ with large $C(\Delta)$.

\begin{tabular}{|c||c|c||c|c|}
\hline \multicolumn{1}{|c||}{} & \multicolumn{2}{c||}{ Composite $\Delta$} & \multicolumn{2}{c|}{ Prime $\Delta$} \\
\hline$p$ & $X$ & $n_{p}$ & $X$ & $n_{p}$ \\
\hline \hline 257 & 396312611459525290 & 30 & 218767904524491586 & 30 \\
277 & 697769695386840996 & 40 & 482626651962422460 & 40 \\
307 & 639546162945216939 & 50 & 762810077127556299 & 50 \\
331 & 390861540221680416 & 60 & 435543163377951528 & 60 \\
\hline
\end{tabular}

such that $\left(\frac{\Delta}{q}\right)=-1$ for all odd primes $q$ less than or equal to $257,277,307,331$, and 353 , for each of the five pairs $\left(A_{p}^{+}, B_{p}\right)$, again sieving only with the primes less than or equal to 229.

For each of the ten sieve jobs, we recorded the first 40 solutions $X$ and computed an estimate of $C(\Delta)$ from (1.1) using only the odd primes less than 300000 . The composite and prime solutions with the largest $C(\Delta)$ estimates for each pair $\left(A_{p}^{-}, B_{p}\right)$ and $\left(A_{p}^{+}, B_{p}\right)$ were selected, and their corresponding $C(\Delta)$ values were approximated to 8 significant digits using (2.9). The values of $X$ yielding the best negative composite and prime solutions are listed in Table 5.2, and those yielding the best positive composite and prime solutions in Table 5.3. In these tables, as well as all subsequent tables, $n_{p}$ denotes the number of decimal digits of the corresponding $|\Delta|$.

In Table 5.4 and 5.5 we present the class numbers, $r(\Delta)$ values, and $C(\Delta)$ approximations for composite and prime $\Delta=-\left(A_{p}^{-}+B_{p} X\right)$, respectively. Tables 5.6 and 5.7 contain the corresponding values for those $\Delta=A_{p}^{+}+B_{p} X$, together with the regulators of the quadratic orders $\mathcal{O}_{\Delta}$. Unfortunately, we have not yet been able to compute $h_{\Delta}$ and $R_{\Delta}$ for the 70-digit positive values of $\Delta$ corresponding to $p=353$, and hence we do not give $C(\Delta)$ values for these two discriminants.

TABLE 5.4. $C(\Delta)$ values for $\Delta=-\left(A_{p}^{-}+B_{p} X\right)$.

\begin{tabular}{|c|r|c|c|}
\hline$p$ & \multicolumn{1}{|c|}{$h_{\Delta}$} & $r(\Delta)$ & $C(\Delta)$ \\
\hline \hline 257 & 31732649150720 & 0.69697563 & 5.24106505 \\
277 & 1976760608074606524 & 0.68146521 & 5.46609410 \\
307 & 61214787639146593755232 & 0.64025219 & 5.37024150 \\
331 & 11759774715020356643576929686 & 0.62718279 & 5.48239469 \\
353 & 2665657958662748945432048763520638 & 0.59889664 & 5.41351382 \\
\hline
\end{tabular}


TABLE 5.5. $C(\Delta)$ values for prime $\Delta=-\left(A_{p}^{-}+B_{p} X\right)$.

\begin{tabular}{|c|r|c|c|}
\hline$p$ & \multicolumn{1}{|c|}{$h_{\Delta}$} & $r(\Delta)$ & $C(\Delta)$ \\
\hline \hline 257 & 32205652661741 & 0.67490844 & 5.07451231 \\
277 & 1616387968869310119 & 0.63738276 & 5.10571428 \\
307 & 121676986663041036395593 & 0.61788587 & 5.19553338 \\
331 & 18644248113618124566660398865 & 0.58425088 & 5.11311211 \\
353 & 508563922487050052185191214201329 & 0.59868248 & 5.38920304 \\
\hline
\end{tabular}

TABLE 5.6. $C(\Delta)$ values for $\Delta=A_{p}^{+}+B_{p} X$.

\begin{tabular}{|c|r|r|c|c|}
\hline$p$ & \multicolumn{1}{|c|}{$h_{\Delta}$} & $R_{\Delta}$ & $r(\Delta)$ & $C(\Delta)$ \\
\hline \hline 257 & 2 & 22720556233553.76096 & 0.70502465 & 5.29857981 \\
277 & 32 & 136748579504713684.06545 & 0.66227548 & 5.32039776 \\
307 & 200 & 1756711054276061939500.58651 & 0.63660975 & 5.36584646 \\
331 & 2 & 13733001618386164806256150133.05014 & 0.61969493 & 5.42321540 \\
\hline
\end{tabular}

TABLE 5.7. $C(\Delta)$ values for prime $\Delta=A_{p}^{+}+B_{p} X$.

\begin{tabular}{|c|r|r|c|c|}
\hline$p$ & \multicolumn{1}{|c|}{$h_{\Delta}$} & \multicolumn{1}{c|}{$R_{\Delta}$} & $r(\Delta)$ & $C(\Delta)$ \\
\hline \hline 257 & 23 & 1486019958907.89109 & 0.69782143 & 5.23353756 \\
277 & 1 & 3524266116230524920.39910 & 0.68457204 & 5.49456617 \\
307 & 1 & 388454242975025771000236.31306 & 0.62860736 & 5.30013214 \\
331 & 3 & 10083301848416825689407861674.34216 & 0.59383948 & 5.19778395 \\
\hline
\end{tabular}

TABle 5.8. Run times for $\Delta=-\left(A_{p}^{-}+B_{p} X\right)$.

\begin{tabular}{|c||r|r|r||c|r|c|}
\hline \multicolumn{1}{|c||}{} & \multicolumn{3}{c||}{ Composite $\Delta$} & \multicolumn{3}{c|}{ Prime $\Delta$} \\
\hline$p$ & $n_{p}$ & \multicolumn{1}{c|}{$t_{h}$} & \multicolumn{1}{c|}{$t_{\text {ver }}$} & $n_{p}$ & \multicolumn{1}{c|}{$t_{h}$} & \multicolumn{1}{c|}{$t_{\text {ver }}$} \\
\hline \hline 257 & 30 & $19.35 \mathrm{~s}$ & $44.26 \mathrm{~s}$ & 30 & $18.79 \mathrm{~s}$ & $44.27 \mathrm{~s}$ \\
277 & 40 & $2.81 \mathrm{~m}$ & $4.12 \mathrm{~m}$ & 39 & $2.77 \mathrm{~m}$ & $4.40 \mathrm{~m}$ \\
307 & 49 & $23.45 \mathrm{~m}$ & $1.58 \mathrm{~h}$ & 49 & $26.34 \mathrm{~m}$ & $2.29 \mathrm{~h}$ \\
331 & 59 & $8.62 \mathrm{~h}$ & $9.15 \mathrm{~h}$ & 60 & $8.26 \mathrm{~h}$ & $8.51 \mathrm{~h}$ \\
353 & 70 & $6.47 \mathrm{~d}$ & $1.18 \mathrm{~s}$ & 69 & $5.29 \mathrm{~d}$ & $0.55 \mathrm{~s}$ \\
\hline
\end{tabular}

We made use of Jacobson's technique [12] to evaluate $h_{\Delta}$ and $R_{\Delta}$ for these large values of $\Delta$. The computations were carried out on a $296 \mathrm{MHz}$ SUN UltraSPARC-II processor with $1024 \mathrm{MB}$ of main memory using $\mathrm{C}++$ routines based on the LiDIA computer algebra library [8]. The $\mathrm{CPU}$ time required for these computations ranged from about 19 seconds to about 6.5 days. In order to guarantee the correctness of our results under the ERH, we also performed the verification described in [12, Ch.3] for each of the ten discriminants. The time required for this additional computation ranged from about 26 seconds to 9 hours. The run-times in CPU seconds (s), minutes $(\mathrm{m})$, hours $(\mathrm{h})$, or days $(\mathrm{d})$ for all $\Delta$ considered above are contained in Tables 5.8 and 5.9. By $t_{h}$ we denote the CPU time required to compute the class 
TABlE 5.9. Run times for $\Delta=A_{p}^{+}+B_{p} X$.

\begin{tabular}{|c||c|c|c||c|c|c|}
\hline \multicolumn{1}{|c||}{} & \multicolumn{3}{c||}{ Composite $\Delta$} & \multicolumn{3}{c|}{ Prime $\Delta$} \\
\hline$p$ & $n_{p}$ & $t_{C l}$ & $t_{\text {ver }}$ & $n_{p}$ & \multicolumn{1}{c|}{$t_{C l}$} & $t_{\text {ver }}$ \\
\hline \hline 257 & 30 & $38.95 \mathrm{~s}$ & $30.48 \mathrm{~s}$ & 30 & $41.42 \mathrm{~s}$ & $25.65 \mathrm{~s}$ \\
277 & 40 & $7.75 \mathrm{~m}$ & $3.50 \mathrm{~m}$ & 40 & $10.02 \mathrm{~m}$ & $3.17 \mathrm{~m}$ \\
307 & 50 & $1.78 \mathrm{~h}$ & $2.25 \mathrm{~h}$ & 50 & $1.92 \mathrm{~h}$ & $1.72 \mathrm{~h}$ \\
331 & 60 & $1.04 \mathrm{~d}$ & $7.84 \mathrm{~h}$ & 60 & $1.97 \mathrm{~d}$ & $4.85 \mathrm{~h}$ \\
\hline
\end{tabular}

number and regulator of $\mathcal{O}_{\Delta}$, and by $t_{v e r}$ the $\mathrm{CPU}$ time required for the $\mathrm{ERH}$ verification.

\section{LaRger $C(\Delta)$ VAlues With FEWER SIEVE MOdULI}

All the examples given above were generated by sieving with odd primes $q \leq 229$. However, the more moduli used, the harder it is to find solutions. For example, in order to generate the 40 solutions $X$ for $A_{331}^{+}+B_{331} X$ it took almost a week of sieve time. Hence, we also ran two sieve jobs using primes $q \leq 199$ in an effort to find $C(\Delta)>5.49456617$, the largest value found using the methods in the previous section. The first of these was designed to generate 70-digit negative discriminants. We used the MSSU to search for values of $X$ such that $\left(\frac{-\left(C_{337}^{-}+D_{337} X\right)}{q_{i}}\right)=-1$ for odd primes $3 \leq q_{i} \leq 199$ and $-\left(C_{337}^{-}+D_{337} X\right) \equiv 5(\bmod 8)$. We used

$$
C_{337}^{-}=1613265, \quad \text { and } \quad D_{337}=\prod_{\substack{q \geq 211 \\ q \text { prime }}}^{337} q,
$$

where $C_{337}^{-}$is the smallest integer such that $\left(\frac{-C_{337}^{-}}{q}\right)=-1$ for all primes $q(211 \leq$ $q \leq 337)$. Thus, every solution $X$ is such that $\left(\frac{-\left(C_{337}^{-}+D_{337} X\right)}{q}\right)=-1$ for all odd primes $3 \leq q \leq 337$, and we only need to sieve with primes less than 200 .

The second sieve job was designed to generate positive discriminants of about 70 decimal digits. We searched for solutions $X$ such that $\left(\frac{C_{337}^{+}+D_{337} X}{q_{i}}\right)=-1$ for all odd primes $3 \leq q_{i} \leq 199$ and $\left.C_{337}^{+}+D_{337} X\right) \equiv 5(\bmod 8) . C_{337}^{+}=14130195$ is the smallest integer such that $\left(\frac{C_{337}^{+}}{q}\right)=-1$ for all primes $q(211 \leq q \leq 337)$. In this case, every solution $X$ is such that $\left(\frac{C_{337}^{+}+D_{337} X}{q}\right)=-1$ for all odd primes $3 \leq q \leq 337$, and as above we only need to sieve with primes less than 200 .

In both cases we generated 500 solutions to the sieve problem, and ordered the solutions according to $C(\Delta)$ estimates computed from (1.1) using only the odd primes less than 300000 . We were able to find these solutions much faster than in the previous problems using sieve moduli up to 229. For the negative discriminants, it took just over 4 days to find the 500 solutions, compared to over a week for only 40 solutions when sieving with $q \leq 229$. The composite and prime solutions with the largest $C(\Delta)$ estimates for each pair $\left(C_{337}^{-}, D_{337}\right)$ and $\left(C_{337}^{+}, D_{337}\right)$ were selected, and their corresponding $C(\Delta)$ values were approximated to 8 significant digits using (2.9). The values of $X$ yielding these best composite and prime values of $\Delta$ are listed in Table 6.1 
TABLE 6.1. $X$ values yielding $\Delta$ with large $C(\Delta)$.

\begin{tabular}{|c||c|c||c|c|}
\hline \multicolumn{1}{|c||}{} & Composite $\Delta$ & \multicolumn{2}{c|}{ Prime $\Delta$} \\
\hline$\Delta$ & $X$ & $n_{p}$ & $X$ & $n_{p}$ \\
\hline \hline$-\left(C_{337}^{-}+D_{337} X\right)$ & 25455834532981358 & 70 & 313761223204200542 & 71 \\
$C_{337}^{+}+D_{337} X$ & 480364831229973862 & 72 & 344681809987259902 & 71 \\
\hline
\end{tabular}

TABLE 6.2. $C(\Delta)$ values for $\Delta=-\left(C_{337}^{-}+D_{337} X\right)$.

\begin{tabular}{|r|r|c|c|}
\hline & \multicolumn{1}{|c|}{$h_{\Delta}$} & $r(\Delta)$ & $C(\Delta)$ \\
\hline \hline$D$ & 3970294065612579776224498944560096 & 0.61158214 & 5.53388912 \\
$p$ & 14171128122001880660726087303711577 & 0.59973638 & 5.44325560 \\
\hline
\end{tabular}

TABle 6.3. $C(\Delta)$ values for $\Delta=C_{337}^{+}+D_{337} X$.

\begin{tabular}{|r|r|c|c|c|}
\hline & $h_{\Delta}$ & $R_{\Delta}$ & $r(\Delta)$ & $C(\Delta)$ \\
\hline \hline$D$ & 4 & 6625291330661652053429358727545606.5573 & 0.62299738 & 5.65726388 \\
$p$ & 3 & 7748091868989848744375988664484659.1689 & 0.60191933 & 5.46368497 \\
\hline
\end{tabular}

TABle 6.4. Run times for $\Delta=-\left(C_{337}^{-}+D_{337} X\right)$ and $\Delta=C_{337}^{+}+D_{337} X$.

\begin{tabular}{|c||c|c|c||c|c|c|}
\hline \multicolumn{1}{|c||}{} & \multicolumn{3}{c||}{ Composite $\Delta$} & \multicolumn{3}{c|}{ Prime $\Delta$} \\
\hline$p$ & $n_{p}$ & \multicolumn{1}{c|}{$t_{h}$} & $t_{\text {ver }}$ & $n_{p}$ & $t_{h}$ & $t_{\text {ver }}$ \\
\hline \hline $337^{-}$ & 70 & $5.84 \mathrm{~d}$ & $3.57 \mathrm{~s}$ & 71 & $5.81 \mathrm{~d}$ & $2.99 \mathrm{~s}$ \\
$337^{+}$ & 72 & $10.68 \mathrm{~d}$ & $7.83 \mathrm{~d}$ & 71 & $7.55 \mathrm{~d}$ & $7.30 \mathrm{~d}$ \\
\hline
\end{tabular}

In Table 6.2 and 6.3 we present the class numbers, regulators, $r(\Delta)$ values, and $C(\Delta)$ approximations for composite and prime $\Delta=-\left(C_{337}^{-}+D_{337} X\right)$ and $\Delta=C_{337}^{+}+D_{337} X$, respectively. In both tables, the entry $D$ denotes the composite discriminant and $p$ indicates the prime discriminant. The CPU time needed on a $296 \mathrm{MHz}$ SUN UltraSPARC-II processor to compute the class numbers and verify them under the ERH are given in Table 6.4

Although we have been able to find significantly larger $C(\Delta)$ values than those in [11], the fact that the $r(\Delta)$ values corresponding to these $\Delta$ are somewhat small suggests that larger $C(\Delta)$ values should be obtainable for other $\Delta$ of the same size. However, as of yet we know of no way to use the MSSU to find $\Delta$ such that $\left(\frac{\Delta}{q}\right)=-1$ for primes $q \leq p$ and $p>229$ without resorting to Lehmer's idea, which unfortunately causes the sizes of $\Delta$ under consideration to increase rapidly. The $\Delta$ presented in [11] are minimal in the sense that they are taken from the set of the smallest integers in absolute value for which $\left(\frac{\Delta}{q}\right)=-1$ for primes $q \leq 199$, and hence the $r(\Delta)$ values are larger than ours, as expected. 
The largest value of $C(\Delta)$ we found is

$$
C(\Delta)=5.65726388
$$

for the 72-digit $\Delta=C_{337}^{+}+D_{337} 480364831229973862$. Thus, according to Conjecture $\mathrm{F}$ and under the assumption of the $\mathrm{ERH}$, we expect the polynomial $x^{2}+x-A$ for $A$ given by

33251810980696878103150085257129508857312847751498190349983874538507313

to have the largest asymptotic density of prime values for any polynomial of this type currently known.

\section{ACKNOWLEDGMEnTs}

The authors wish to thank an anonymous referee for several helpful suggestions for improving the paper, as well as the Centre for Applied Cryptographic Research at the University of Waterloo for their support and for the use of their computing facilities.

\section{REFERENCES}

1. C.S. Abel, Ein Algorithmus zur Berechnung der Klassenzahl und des Regulators reellquadratischer Ordnungen, Ph.D. thesis, Universität des Saarlandes, Saarbrücken, Germany, 1994.

2. E. Bach, Improved approximations for Euler products, Number Theory: CMS Proc., vol. 15, Amer. Math. Soc., Providence, RI, 1995, pp. 13-28. MR 96i:11124

3. N.G.W.H. Beeger, Report on some calculations of prime numbers, Nieuw Archief voor Wiskunde (2) 20 (1939), 48-50. MR 1:65g

4. J. Buchmann, A subexponential algorithm for the determination of class groups and regulators of algebraic number fields, Séminaire de Théorie des Nombres (Paris), 1988-89, Birkhäuser, Boston, 1990, pp. 27-41. MR 92g:11125

5. H. Cohen, F. Diaz y Diaz, and M. Olivier, Calculs de nombres de classes et de régulateurs de corps quadratiques en temps sous-exponentiel, Séminaire de Théorie des Nombres (Paris), 1990-91, Birkhäser, Boston, 1993, pp. 35-46. MR 94m:11151

6. S. Düllmann, Ein Algorithmus zur Bestimmung der Klassengruppe positiv definiter binärer quadratischer Formen, Ph.D. thesis, Universität des Saarlandes, Saarbrücken, Germany, 1991.

7. G.W. Fung and H.C. Williams, Quadratic polynomials which have a high density of prime values, Math. Comp. 55 (1990), 345-353. MR 90j:11090

8. The LiDIA Group, LiDIA: a c++ library for computational number theory, Software, Technische Universität Darmstadt, Germany, 1997, See http://www.informatik.tu-darmstadt.de/TI/LiDIA.

9. J.L. Hafner and K.S. McCurley, A rigorous subexponential algorithm for computation of class groups, J. Amer. Math. Soc. 2 (1989), 837-850. MR 91f:11090

10. G.H. Hardy and J.E. Littlewood, Partitio numerorum III: On the expression of a number as a sum of primes, Acta Math. 44 (1923), 1-70.

11. M.J. Jacobson, Jr., Computational techniques in quadratic fields, Master's thesis, University of Manitoba, Winnipeg, Manitoba, 1995.

12. $ـ$ Subexponential class group computation in quadratic orders, Ph.D. thesis, Technische Universität Darmstadt, Darmstadt, Germany, 1999.

13. P.T. Joshi, The size of $L(1, \chi)$ for real nonprincipal residue characters $\chi$ with prime modulus, J. Number Theory 2 (1970), 58-73. MR 40:4215

14. D.H. Lehmer, On the function $x^{2}+x+A$, Sphinx 6 (1937), 212-214, 1936 and 7:40.

15. D.H. Lehmer, E. Lehmer, and D. Shanks, Integer sequences having prescribed quadratic character, Math. Comp. 24 (1970), no. 110, 433-451. MR 42:5889

16. J.E. Littlewood, On the class number of the corpus $P(\sqrt{ }-k)$, Proc. London Math. Soc. 27 (1928), 358-372.

17. R.F. Lukes, A very fast electronic number sieve, Ph.D. thesis, University of Manitoba, Winnipeg, Manitoba, 1995. 
18. R.F. Lukes, C.D. Patterson, and H.C. Williams, Numerical sieving devices: Their history and some applications, Nieuw Archief voor Wiskunde (4) 13 (1995), 113-139. MR 96m:11082

19. R.A. Mollin and H.C. Williams, Computation of the class number of a real quadratic field, Utilitas Mathematica 41 (1992), 259-308. MR 93d:11134

20. S. Paulus, An algorithm of subexponential type computing the class group of quadratic orders over principal ideal domains, Algorithmic Number Theory - ANTS-II (Université Bordeaux I, Talence, France), Lecture Notes in Computer Science, vol. 1122, Springer-Verlag, Berlin, 1996, pp. 243-257. MR 98c:11143

21. L. Poletti, Il contributo italiano alla tavola dei numeri primi, Rivista di Matematica della Università di Parma 2 (1951), 417-434. MR 14:121d

22. M. Seysen, A probabilistic factorization algorithm with quadratic forms of negative discriminant, Math. Comp. 48 (1987), 757-780. MR 88d:11129

23. D. Shanks, On the conjecture of Hardy and Littlewood concerning the number of primes of the form $n^{2}+a$, Math. Comp. 14 (1960), 320-332. MR 22:10960

24. R.D. Silverman, The multiple polynomial quadratic sieve, Math. Comp. 48 (1987), 329-339. MR 88c:11079

Department of Computer Science, University of Manitoba, Winnipeg, Manitoba, CANADA R3T 2N2

E-mail address: jacobs@cs.umanitoba.ca

Department of Mathematics and Statistics, MS 360, 2500 University Drive N.W., University of Calgary, Calgary, Alberta, Canada T2N 1N4

E-mail address: williams@math.ucalgary.ca 